\section{Revista de la \\ Universidad del Thulia}

Fundada en 1947 por el Dr. Jesúns Enrique Lossada

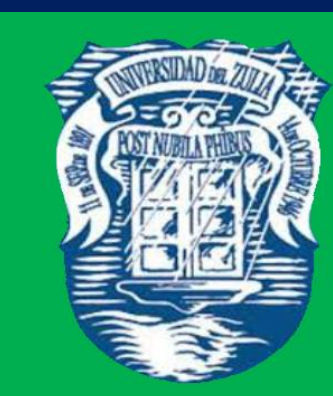

Ciencias del

Algreo

Ingemieria

y Teemología

\section{Aกัต 11 No 29}

Enero - Abril 2021

Tercera Época

Maracaibo-Venezuela 


\title{
Convergencia a NIIF: impactos en estructura financiera de los fondos de empleados del Departamento de Sucre - Colombia
}

\author{
Jaime Guevara Sanabria* \\ Miguel Tamara Contreras** \\ Rafael Buelvas Peralta**
}

RESUMEN

En la presente investigación se evaluó el efecto de aplicar las Normas Internacionales de Información Financiera para Pymes en los fondos de empleados del Departamento de Sucre - Colombia, mediante la comparación de los principales indicadores financieros tanto para la norma internacional como en la norma local colombiana. Para lograr la comparación de las dos normas contables y para evitar los problemas de desempeño de los fondos, se tomaron los estados financieros al 31 de diciembre de 2014 bajo norma colombiana y el estado financiero para propósitos de conversión bajo norma internacional al 1 de enero de 2015. Adicionalmente se realizó una modelación para medir el impacto de la norma original NIC 32, en donde se reclasificaron los aportes sociales al pasivo. La investigación fue de carácter descriptiva, y para ello se tomó como base la información reportada por los fondos a la Superintendencia de la Economía Solidaria. Dentro de las principales conclusiones se encuentra que no se visualizan impactos significativos en la estructura financiera de los fondos de empleados de Sucre por el cambio de regulación contable hacia NIIF.

PALABRAS CLAVE: Fondos de empleados; Normas Internacionales de Información Financiera; convergencia; indicadores financieros; aportes sociales

*Contador Público. Profesor de la Universidad de Antioquia, Colombia, jalberto.guevara@udea.edu.co

**Contador Público, con Maestría en Ciencias Contables.

Recibido: 13/11/2019

Aceptado: 18/12/2019 
REVISTA DE LA UNIVERSIDAD DEL ZULIA. $3^{a}$ época. Año $11 \mathrm{~N}^{\circ}$ 29, 2020

Jaime Guevara et al. /// Convergencia a NIIF: impactos en estructura financiera ...

\section{Convergence to IFRS: impacts on financial structure of employee funds of Sucre Department - Colombia}

ABSTRACT

In the present investigation, the effect of applying the International Financial Reporting Standards for SMEs in the employee funds of the Department of Sucre - Colombia was evaluated, by comparing the main financial indicators for both the international standard and the Colombian local standard. To achieve the comparison of the two accounting standards and to avoid the performance problems of the funds, the financial statements were taken as of December 31, 2014 under Colombian standard and the financial statement for conversion purposes under international standard as of January 1, 2015. Additionally, a modeling was carried out to measure the impact of the original IAS 32 standard, where social contributions to the liabilities were reclassified. The investigation was descriptive, and the information reported by the funds to the Superintendence of the Solidarity Economy was taken as a basis. Among the main conclusions is that there are no significant impacts on the financial structure of Sucre employee funds due to the change in accounting regulation towards IFRS.

KEY WORDS: Employee funds; International Financial Reporting Standards; convergence; financial indicators; social contributions

Introducción

La convergencia de normas locales en Colombia, hacia Normas Internacionales de información financiera (en adelante NIIF), ha generado diferentes reacciones entre los sectores y usuarios de la información financiera, debido entre otros, a los cambios que estas generan en las bases de reconocimiento, medición y revelación con respecto al GAAP local, pudiendo alterar la estructura financiera y por ende los diferentes indicadores económicos (Arias y Sánchez 2014).

Este escenario no es ajeno a las empresas del sector solidario, que a partir l de enero de 2015 deben converger en forma obligatoria a NIIF, de acuerdo a la| Circular Externa 5 de 2014 de la Superintendencia de Economía Solidaria. Por ser los Fondos de Empleados entidades de carácter social y solidario que propende por satisfacer las necesidades colectivas de sus asociados y desde luego, preservar los aportes de los mismos, "las cooperativas y en general, las entidades del sector solidario, como las asociaciones 
REVISTA DE LA UNIVERSIDAD DEL ZULIA. 3época. Año 11 N² 29, 2020 Jaime Guevara et al. /// Convergencia a NIIF: impactos en estructura financiera ...

mutuales, han adquirido recientemente gran relevancia por su contribución en la economía

del país, toda vez que estas instituciones son consideradas un elemento importante de desarrollo social, económico, cultural y ambiental” Morales, M. (2014 p.132)

Sin embargo, el sector cooperativo es el que levantó todo el revuelo, ya que originalmente la NIC 32 obligaba a que los aportes de los socios no se contabilizaran como capital social de la cooperativa, sino que dichos aportes deberían ser llevados como pasivos, porque existe un derecho del socio para, en caso de retiro, reclamar su reembolso, golpeando la estructura del patrimonio de las cooperativas (Álvarez B. y Suarez E. 2015). Finalmente, la norma colombiana decidió no aplicar este lineamiento de NIC 32, y dejar los respectivos aportes de los socios en el patrimonio. En este sentido bajo una metodología de investigación descriptiva, se pretende describir los posibles efectos del cambio a NIF, incluyendo la modelación con los preceptos originales de NIC 32.

La estructura del artículo empieza por describir algunos de los principales impactos a nivel mundial por la implementación de NIIF en el mundo y luego especificándolos en la economía solidaria, para continuar con la descripción del sector de la economía solidaria en Colombia finalizando con el análisis de los impactos en los indicadores financieros seleccionados por la implementación de NIIF, incluyendo la modelación por la aplicación de NIC 32.

\section{Metodología}

Este trabajo se desarrolla con una metodología de tipo descriptiva, ya que el análisis de la información lleva a cabo un análisis estructural, entendido como el estudio que permite tener una panorámica general de los distintos componentes de los estados financieros, es decir, el comportamiento de los elementos que constituyen cada uno de esos componentes y que se identifican como activos, pasivos, patrimonio, gastos e ingresos. Además, "la descripción científica se basa en el propósito de dar a conocer una información, un hecho, simplemente un dato, tal cual es, depurando al máximo las apreciaciones subjetivas del sujeto" (Cerda, 2002, p.75). Para lograr la medición de impactos por el cambio a NIIF, se tomaron los balances de transmisión a la superintendencia de economía 
REVISTA DE LA UNIVERSIDAD DEL ZULIA. 3época. Año 11 N² 29, 2020 Jaime Guevara et al. /// Convergencia a NIIF: impactos en estructura financiera ...

solidaría al 31 de diciembre de 2014 bajo (COLGAAP) y el estado de situación financiera de apertura para propósitos de conversión bajo NIIF (ESFA) al 1 de enero de 2015, de los fondos de empleados del Departamento de Sucre.

Para la medición de los impactos se realizaron análisis financiero, de los dos estándares a la misa fecha de corte, lo anterior para eliminar incongruencias en los resultados de los indicadores debido desempeño de las cooperativas. Los indicadores financieros utilizados son de liquidez, estructura y endeudamiento, cuyos resultados son comparados por los arrojados bajo COLGAAP y NIIF. Adicionalmente, para el análisis de los impactos de NIC 32 se realizaron una modelación, en donde se reclasificaron los aportes sociales al pasivo.

\section{Evolución de las NIIF a nivel mundial}

Una de las intenciones de los reguladores contables con la implementación de NIIF, es homologar las políticas contables, con el propósito de reducir problemas de comparabilidad, adicionalmente se pretende mejorar la calidad de la información contable, situación que se ha logrado con el proceso de implementación (Barth Landsman y Lang, 2008). Este proceso se inició en el año 1973, por iniciativa de algunos países europeos, sin embargo, la aceptación de las NIIF por parte de muchos países depende de la aceptación de los mercados de valores, situación que fortalecerá la expansión de las NIIF a nivel mundial. (Restrepo 2011).

De esta forma las NIIF han adquirido una verdadera connotación internacional, principalmente por el apoyo de instituciones como la Comunidad Económica Europea, el G20 y la Organización Internacional de Comisiones de Valores IOSCO. Inclusive la bolsa de valores de Nueva York (NYSE) permitió la presentación de los estados financieros bajo NIIF para los emisores foráneos. Es así como las recientes convergencias hacia las NIIF, que han sido llevadas a cabo por más de 100 países, se convierten en el hito más importante de los cambios de regulación en la historia de la contabilidad (Dasker, Hail, Leuz \& Verdi, 2008). 
REVISTA DE LA UNIVERSIDAD DEL ZULIA. 3época. Año 11 N² 29, 2020 Jaime Guevara et al. /// Convergencia a NIIF: impactos en estructura financiera ...

Algunos de los estudios sobre el proceso de implementación de NIIF muestran resultados mixtos sobre la calidad e impactos sobre los estados financieros, se destacan el trabajo de Callao, S., Ferrer, C., Jarne, J., y Laínez, J. (2010) en el Reino Unido y en España, concluyeron que la aplicación de NIIF afectó negativamente la relevancia de los reportes financieros de estos países, entre otros, por la incapacidad de identificar los valores de mercado en los estados financieros de las distintas compañías. Por otro lado, se encuentran estudios como el de Pérez, R., Urquía, E. y Muñoz, C. (2009) acerca de la relación de éxito que tienen las pymes que se interesan por la implementación de las NIIF en España, por la connotación de que en ese país la implementación de NIIF para Pymes es voluntaria.

En Latinoamérica, las pymes venezolanas, Zapata, G., Hernández, A. (2010) indicaron que la problemática de implementación, gira alrededor de la norma tributaria y del cómo evitar sanciones más que en la línea de aspectos financieros o administrativos; lo anterior se evidencia por la estructura administrativa que tienen las pyme sque es diferente a la de las empresas que cotizan en bolsa. Mientras tanto, Lima, R. (2007) argumenta que las pymes son un importante grupo de empresas que recoge la mayoría de las organizaciones, por lo que desde la academia se han tomado decisiones para profundizar más en las pymes y con ello fortalecer este sector de la economía e influir en instaurar normas contables que vayan acordes con las características que estas tienen.

Las variedades de estudios pasan también con la implementación por primera vez, es así como el trabajo realizado por Vásquez, N. (2013) en México sobre la NIIF 1, evidencian que las excepciones obligatorias que más aplicaron las empresas fueron las relacionadas con: estimaciones, contabilidad de coberturas y participaciones no controladoras.

En Chile, Silva, B. y Garrido, C. (2006) concluyen que la implementación de NIIF, busca ser el insumo para inversionistas de cualquier lugar del mundo para poder tomar adecuadas decisiones y así traer inversión extranjera. Adicionalmente, según Yáñez, V, Pilar, F. e Inestrosa, C. (2010), el proceso de implementación de NIIF implica para el profesional contable y financiero un desarrollo mucho más profundo de sus competencias y habilidades profesionales. 
REVISTA DE LA UNIVERSIDAD DEL ZULIA. 3época. Año 11 N² 29, 2020 Jaime Guevara et al. /// Convergencia a NIIF: impactos en estructura financiera ...

Uno de los estudios más amplios de implementación de NIIF, realizados en Latinoamérica, incluyendo a Colombia, es el realizado por Vásquez, N., Carril, M. y Pascual, M. (2013) en donde refuerzan que uno de los factores que influye para la adopción de NIIF es la existencia de una bolsa de valores fuerte, debido a la necesidad de los usuarios de la información financiera confiable.

Entretanto, Cañibano, L. y Gisbert, A. (2007) presentan un panorama de la obligatoriedad de algunos países vs el carácter voluntario y su impacto en las grandes empresas que cotizan en bolsa, mostrando que se mejora la presentación de información financiera bajo NIIF.

Finalmente, se encuentran trabajos como el de Torres, F. y Rodríguez, B. (2008) que evidencia más consecuencias desde el punto de vista conceptual y teórico, como el impacto de la nueva información financiera para su uso por parte de los distintos usuarios de la información, sin trascender al análisis de casos o eventos prácticos de aplicación de estándares en alguna temática cercana a las pymes del país.

En cuanto al proceso de adopción de NIIF en Colombia, a diferencia de países como los europeos, ha sido lento en la actualización de la normatividad contable, en especial, por parte del Consejo Permanente para la Evaluación de las Normas sobre Contabilidad, retrasando la incorporación a diversos tratados internacionales que exigen la preparación de estados financieros rigurosos, consistentes con indicadores de alta calidad y transparencia.

\section{Impacto de las NIIF en el sector de la economía solidaria}

La primera gran preocupación de la aplicación de NIIF para el sector solidario, es que las estas normas no pueden ser tomadas como base para la aplicación a los diferentes tipos de industrias o de sectores de la economía (Gómez, 2004), recordando que ni las sociedades cooperativas, son entidades que coticen en mercados bursátiles. De esta forma su implementación puede traer impactos financieros importantes de no tomarse un proceso serio de adaptación de las normas a la economía solidaría. (Castaño, C., Zamarra, J. y Correa, J., 2014) Estas incertidumbres se encuentran también en varios escritos de 
REVISTA DE LA UNIVERSIDAD DEL ZULIA. 3época. Año 11 N² 29, 2020 Jaime Guevara et al. /// Convergencia a NIIF: impactos en estructura financiera ...

carácter internacional, siendo la más importante de ellas la clasificación de las aportaciones sociales realizadas por los socios como pasivo financiero, cambiando así la actual estructura de capital social de las cooperativas ya que dichos aportes se contabilizan como patrimonio. A nivel nacional Rendón, B., Montaño, E. Gaitán, G. (2013) y Correa, J. y Ojeda, N. (2014), en sus investigaciones evalúan el efecto de aplicar la norma internacional de contabilidad (NIC 32), especialmente en lo relacionado con instrumentos financieros y los aportes sociales reconocidos y revelados por las entidades cooperativas redundando en el efecto de la estructura patrimonial de las mismas. Lo anterior ha generado mucha sensibilidad en el sector cooperativo y especialmente a los responsables de estas empresas ya que la estructura, composición del capital social y de los fondos propios a la luz de las NIIF cambiaría, generando un giro de ciento ochenta grados en su calificación patrimonial. (Polo, F., Cubedo, M., 2007); (Molina, R. 2007). La interpretación CINIC2 queda corta ante esta problemática ya que el reembolso de las aportaciones al capital social en caso de retiro del socio (implicando una reducción del capital) es una de las características de las cooperativas (Vargas Vasserot, 2007).

En este mismo sentido, Martínez, F. (2014), refiriéndose al caso de las cooperativas panameñas, deja sentada su preocupación de buscar un modelo, que sea propio a las cooperativas en cuanto a la implementación de las NIIF, este nuevo modelo debe desarrollar nuevas mediciones de instrumentos financieros ya que las exigibles por NIIF no aplican al sector, ya que han sido desarrolladas para el mercado de capitales. Así mismo, las sociedades cooperativas están jugando un rol importante en el proceso de convergencia contable internacional, en las cuales deberían tener en cuenta la realidad del sector con los principios y valores fundamentales como los solidarios (Alianza Cooperativa Internacional para las Américas, ACI-Américas, 2012).

Otro aspecto importante según Castaño, C., Correa, J. y Zamarra J. (2014) en el sector solidario es el cálculo de deterioro de la cartera, que se determina de acuerdo con el tiempo vencido a la tasa de referencia, contabilizándose el mencionado deterioro, mediante una provisión, según el mecanismo de la administración de riesgo crediticio (SARC). El estándar internacional exige que al final de cada periodo, se debe realizar una evaluación 
REVISTA DE LA UNIVERSIDAD DEL ZULIA. 3ㄹe época. Año 11 N² 29, 2020 Jaime Guevara et al. /// Convergencia a NIIF: impactos en estructura financiera ...

para determinar la provisión por deterioro de cartera, medida al costo o al costo amortizado. Ahora, al entrar en vigencia el SARC, quedaría eliminada la clasificación por edades de acuerdo con la Circular Básica Contable y Financiera, no obstante, se podría presentar la cartera en una condición de mayor riesgo, de acuerdo a los criterios señalados en la norma. Para Cardozo, H. (2015), las normas o estándares internacionales obligan a establecer el deterioro individual de cada deudor mitigando la posibilidad de existir alguna contingencia de pérdida, en donde la entidad solidaria debe dejar evidencia en un formato especial en la cédula de cada deudor.

Por todo lo expuesto y ante solicitudes expresadas por la Superintendencia de la Economía Solidaria, en Colombia, se contempla una salvedad en la aplicación de las NIIF en relación con el tratamiento y deterioro de la cartera de las entidades sujetas a su inspección y vigilancia (NIC 39 y NIIF 9), el Consejo Técnico de Contaduría Pública (C.T.C.P), plantea que, dado que las estructuras financieras y de negocios de las cooperativas de ahorro y crédito vigiladas por la S.E.S., son similares a las cooperativas financieras y a las compañías de financiamiento, se le dé el mismo tratamiento señalado en el Decreto 1851 de 2013 para las entidades de crédito vigiladas por la Superintendencia Financiera, en aras de la equidad, que luego de un análisis de impacto de la aplicación de las NIIF en este sector solidario, el día 27 de julio de 2015, el (C.T.C.P), emitió el documento, llamado propuesta de excepción a la aplicación integral de las NIIF en el tratamiento de la cartera de crédito de las entidades vigiladas por la S.E.S., en la cual determina que es viable el establecimiento de una excepción en los estados financieros individuales o separados que permita a la S.E.S. mantener el modelo de provisión actual de la cartera de préstamos, mientras se implementa el modelo de administración de riesgo crediticio.

\section{3.l. Economía solidaria en Colombia}

Según los datos de la Superintendencia de Economía Solidaria., se estima que en Colombia el sector solidario cerró en 2014 con 4.708 entidades vigiladas, 5,67\% menos que 
REVISTA DE LA UNIVERSIDAD DEL ZULIA. 3época. Año 11 N² 29, 2020 Jaime Guevara et al. /// Convergencia a NIIF: impactos en estructura financiera ...

en 2013. Según Cenicoop y Confecoop, la principal explicación a la reducción del número de entidades fue la salida de algunas cooperativas de trabajo asociados, debido a la implementación de medidas por parte de los entes de control con el fin de regular el uso indebido de este modelo cooperativo.

Del total de entidades que reportaron información en 2014, las más importantes son las cooperativas con el 57,3\% y los fondos de empleados con el 35\%. Véase (Tabla 1).

Las Cooperativas de Ahorro y Crédito que son solo el 4\% tienen el mayor número de asociados con el 42\%, sin embargo, generan menos empleos que las Cooperativas que con $42 \%$ de los asociados tienen el $37 \%$ de empleados.

En cuanto al tamaño, las mayores participaciones son las cooperativas con activos que representa el $43 \%$ del total, y a su vez son las que tienen mayores ingresos con $87 \%$. En resultados las cooperativas también son las de mayores excedentes con el 41\%; en cuanto a los excedentes resaltamos el comportamiento de los fondos de empleados que a pesar de tamaño pequeño (20\%) generan excedentes que equivalen al $21 \%$ del sector.

Tabla II. Cifras Economía solidaria Colombia, 2004

(en millones de pesos)

\begin{tabular}{lrrrrr}
\hline \multicolumn{1}{c}{ Categoría } & Activo & Pasivo & Patrimonio & Ingresos & $\begin{array}{c}\text { Excedentes } \\
\text { y/o } \\
\text { pérdidas }\end{array}$ \\
\hline Cooperativas & 12.439 .863 & 5.950 .508 & 6.489 .355 & 15.464 .610 & 213.211 \\
$\begin{array}{l}\text { Cooperativas de Ahorro } \\
\text { y Crédito }\end{array}$ & 10.037 .428 & 6.199 .974 & 3.837 .454 & 1.465 .141 & 198.350 \\
Fondos de Empleados & 6.403 .644 & 4.350 .745 & 2.052 .899 & 771.144 & 110.986 \\
Asociaciones Mutuales & 173.605 & 123.341 & 50.264 & 47.676 & -673 \\
Otras Organizaciones & 30.820 & 6.607 & 24.213 & 44.448 & 717 \\
\hline Total & 29.085 .360 & 16.631 .175 & 12.454 .185 & 17.793 .019 & 522.592 \\
\hline
\end{tabular}

Fuente: Elaboración propia. 
REVISTA DE LA UNIVERSIDAD DEL ZULIA. 3época. Año 11 N²9, 2020 Jaime Guevara et al. /// Convergencia a NIIF: impactos en estructura financiera ...

\subsection{Fondos de empleados}

Los fondos de empleados son empresas asociativas, de derecho privado, sin ánimo de lucro, constituidas por trabajadores dependientes y subordinados en donde la asociación y el retiro son voluntarios.

Cardozo, H. (2008) expresa que, "la disparidad de los Fondos de Empleados con las instituciones que ejercen actividad financiera radica en que sus asociados capitalizan su empresa, regularmente, a través de descuentos de nómina, dándole permanente liquidez a su función de intermediación, a la vez que reduce sus riesgos. Además, los Fondos de Empleados pueden imponer sanciones, incluso de orden social, a sus asociados, lo cual le da una ventaja sobre los bancos comerciales" (p.2). Dentro de las ventajas de los Fondos de Empleados, existe el hecho de estar cerca del trabajador y conocer sus problemas y necesidades. A diferencia de las entidades financieras que desconocen aspectos claves de la situación de sus clientes, generando asimetría en la información.

En Colombia, están registrados 1.646 fondos de empleados con 976.466 asociados los cuales generan 17.000 empleos aproximadamente, con un ratio de un empleado por cada 56 asociados, en la región Caribe este ratio parece excesivo, con una relación de un empleado por cada 22 asociados, a diferencia de la región Pacífica que tiene por cada 94 asociados un empleado.

La Región Caribe ocupa el tercer lugar en cuanto a presencia de fondos de empleados, con una participación en activos de $\$ 400.162$ millones, siendo el 6,2\%, del total país, en cuanto a excedentes reportan $\$ 10.524$ millones y participan con el 9,48\%. Dentro de la región Caribe el Departamento del Atlántico es el líder en cuanto a participación por tamaño de los fondos con el 65\%.

Destacamos la relación activo-excedentes que muestra Sucre ya que posee el triple de excedentes que el Magdalena con la misma cantidad de activos, y el 18\% más de excedentes que Córdoba que tiene el doble de activos. También es de anotar que, los fondos del Atlántico necesitan menor inversión de activo para generar excedentes. 
REVISTA DE LA UNIVERSIDAD DEL ZULIA. 3época. Año 11 N²9, 2020 Jaime Guevara et al. /// Convergencia a NIIF: impactos en estructura financiera ...

Tabla III. Fondos de empleados, Regiones 2014

\begin{tabular}{lrrrrrr}
\hline Región & $\begin{array}{c}\text { \# Fondos } \\
\text { empleados }\end{array}$ & \multicolumn{1}{l}{$\%$} & Asociados & $\%$ & Empleados & $\%$ \\
\hline Amazonía & 6 & $0,4 \%$ & 1.232 & $0,1 \%$ & 12 & $0,1 \%$ \\
Andina & 1.247 & $75,8 \%$ & 770.285 & $78,6 \%$ & 13.460 & $77,3 \%$ \\
Caribe & 121 & $7,4 \%$ & 50.750 & $5,2 \%$ & 2.276 & $13,1 \%$ \\
Orinoquía & 12 & $0,7 \%$ & 4.010 & $0,4 \%$ & 50 & $0,3 \%$ \\
Pacífica & 260 & $15,8 \%$ & 153.189 & $15,6 \%$ & 1.625 & $9,3 \%$ \\
\hline Total & 1.646 & $100 \%$ & 979.466 & $100 \%$ & 17.423 & $100 \%$ \\
\hline
\end{tabular}

Fuente: Elaboración propia.

Tabla IV. Resultados financieros de Fondos de Empleados, región Caribe, 2014

(en millones de pesos)

\begin{tabular}{llrrrr}
\hline \multicolumn{1}{c}{ Departamento } & Activo & Pasivo & Patrimonio & Ingresos & $\begin{array}{c}\text { Excedentes y/o } \\
\text { pérdidas }\end{array}$ \\
\hline Atlántico & $\$ 260.922$ & $\$ 150.423$ & $\$ 110.499$ & $\$ 32.044$ & $\$ 7.571$ \\
Bolívar & $\$ 84.779$ & $\$ 63.700$ & $\$ 21.079$ & $\$ 9.681$ & $\$ 1.706$ \\
Cesar & $\$ 28.814$ & $\$ 12.465$ & $\$ 16.349$ & $\$ 3.400$ & $\$ 472$ \\
Córdoba & $\$ 12.539$ & $\$ 6.255$ & $\$ 6.284$ & $\$ 2.475$ & $\$ 287$ \\
Sucre & $\$ 5.763$ & $\$ 1.559$ & $\$ 4.203$ & $\$ 902$ & $\$ 351$ \\
Magdalena & $\$ 5.327$ & $\$ 2.400$ & $\$ 2.927$ & $\$ 663$ & $\$ 112$ \\
Guajira & $\$ 2.018$ & $\$ 670$ & $\$ 1.348$ & $\$ 273$ & $\$ 26$ \\
\hline Total & $\$ 400.162$ & $\$ 237.472$ & $\$ 162.689$ & $\$ 49.438$ & $\$ 10.524$ \\
\hline
\end{tabular}

Fuente: Elaboración propia. 
REVISTA DE LA UNIVERSIDAD DEL ZULIA. 3época. Año 11 N² 29, 2020 Jaime Guevara et al. /// Convergencia a NIIF: impactos en estructura financiera ...

En cuento a los fondos de empleados del Departamento de Sucre, para el año 2014, cuenta con 5 fondos al cual pertenecen 645 asociados, generado 8 empleos directos con un ratio de 80 asociados por empleo. Poseen activos totales por $\$ 5.763$ millones y excedentes de $\$ 351$ millones. (Tabla 7).

Destacamos que FEHORES tiene el $71 \%$ más de excedentes que FEPEC que tiene $51 \%$ más de activos; y tiene aproximadamente los mismos excedentes que LICAPEFE que cuenta con el 25\% más de activos, sin embargo, el FEUS es el fondo más importante en todas sus variables.

Además, se observa que el activo de FEHORES es financiado el 63\% por el pasivo, debido a que gran parte de la cuota periódica obligatoria es destinada a depósito de ahorro permanente, más los ahorros voluntarios. Caso contrario ocurre con FEDECATOL, FEUS, FEPEC y LICAPEFE donde el patrimonio financia el 61,55\%, 82,48\%, 72,94\% y 63,89\%, respectivamente.

Tabla V. Fondos de Empleados del departamento de Sucre, 2014 (millones de pesos)

\begin{tabular}{lrrrrrrrr}
\hline \multicolumn{1}{c}{ Sigla } & Asociados & Empleados & Activo & Pasivo & Patrimonio & Ingresos & $\begin{array}{c}\text { Excedentes } \\
\text { y/o } \\
\text { Perdidas }\end{array}$ \\
\hline FEDECATOL & 93 & 1 & $\$ 1.056$ & $\$ 407$ & $\$ 650$ & $\$ 148$ & $\$ 68$ \\
FEUS & 285 & 4 & $\$ 3.225$ & $\$ 565$ & $\$ 2.660$ & $\$ 500$ & $\$ 209$ \\
FEHORES & 103 & 1 & $\$ 393$ & $\$ 248$ & $\$ 145$ & $\$ 66$ & $\$ 29$ \\
FEPEC & 106 & 1 & $\$ 595$ & $\$ 161$ & $\$ 434$ & $\$ 126$ & $\$ 17$ \\
LICAPEFE & 58 & 1 & $\$ 493$ & $\$ 178$ & $\$ 315$ & $\$ 61$ & $\$ 28$ \\
\hline Total & 645 & 8 & 5.763 & 1.559 & 4.203 & 902 & 351 \\
\hline
\end{tabular}

Fuente: Elaboración propia. 
REVISTA DE LA UNIVERSIDAD DEL ZULIA. 3ㄹe época. Año 11 N² 29, 2020 Jaime Guevara et al. /// Convergencia a NIIF: impactos en estructura financiera ...

\section{Análisis financiero NIIF - Col GAAP y Modelación}

En un entorno competitivo para la empresa de hoy, independientemente del sector al que pertenezca, es importante conocer su situación financiera para tomar decisiones que le permitan alcanzar sus objetivos y metas fijadas. Por lo que no es suficiente contar solo con la información contable, sino que se requiere interpretarla y analizarla para entender el origen y comportamiento de los recursos de la empresa; es por esto, que surge la necesidad del análisis financiero. De acuerdo a García, O. (1999), Castaño, C. y Arias, J. (2013) y Correa, J y otros (2010), el diagnóstico financiero o análisis financiero comprende el estudio e interpretación de la información proporcionada por la contabilidad, e inclusive toda la información disponible, contextualizada y estructural, que sea cuantitativa y cualitativa, histórica y proyectada, para tratar de determinar la situación económicofinanciera de la empresa o de un área específica de ésta, haciendo uso de técnicas, entre otras, de los indicadores financieros para llegar a conclusiones más complejas en un entorno dinámico de la empresa.

Para el caso concreto de los Fondos de Empleados y de acuerdo a la Circular Contable y Financiera Nº04 de 2008, el análisis financiero para una organización solidaria, debe evaluar el desempeño financiero y operacional, es necesario la aplicación de indicadores, lo que permitirá a los administradores tomar decisiones en cualquier momento del ejercicio contable. Según García, O., Legis Editores S.A. (2015), los indicadores financieros de propósito especial, permiten conocer aspectos como la liquidez, rotación, solvencia, rentabilidad y endeudamiento del ente evaluado.

En este capítulo se describen los resultados de los indicadores financieros bajo COLGAAP y NIIF, aplicable en Colombia a los fondos de empleados del Departamento de Sucre, tanto par el riesgo de liquidez, cartera, financiero, estructura y operacional, con el propósito de observar el resultado bajo Colgaap y por la convergencia a NIIF.

Adicionalmente se describe los resultados de la modelación realizada por los autores, que consiste en el re cálculo de los indicadores financieros, tomando como base las NIIF 
REVISTA DE LA UNIVERSIDAD DEL ZULIA. 3época. Año 11 N² 29, 2020 Jaime Guevara et al. /// Convergencia a NIIF: impactos en estructura financiera ...

FULL, es decir considerando entre otros los aportes sociales como pasivo y no como patrimonio.

Los indicadores aplicables a los fondos de empleados, hacen uso de umbrales que reflejan la presencia o ausencia de amenazas que afectan el nivel de salud financiera, y se presentan como indicadores de color. Los tres colores que permite la Superintendencia de Economía Solidaria son: el verde, el amarillo, y rojo. El verde indica que la entidad tiene vía libre para desarrollar sus actividades, y se considera como buena "salud" financiera, el color amarillo indica precaución y análisis de probables situaciones que afectan el desarrollo de las actividades y el color rojo, indica posee problemas en la viabilidad o desarrollo de sus actividades, es decir tiene amenazas contundentes o definitivamente cesa de sus intenciones operacionales

\subsection{Indicadores de Riesgo de Liquidez}

Según los resultados arrojados por los indicadores de riesgo de liquidez, los fondos en estudio están dentro del umbral VERDE en el fondo de liquidez y el nivel de disponible e inversiones, en los tres análisis. (Véase tabla VI). Hay una variación en número de veces en razón corriente, en prueba ácida y solidez por la reclasificación de las cuentas convenios por cobrar, intereses, responsabilidades pendientes. Al implementar las NIIF la capacidad para responder a sus obligaciones a corto plazo ha disminuido, por ejemplo, en razón corriente bajo COLGAAP los fondos contaban con $\$ 3,40$ por $\$ 1$ adeudado al corto plazo como respaldo de sus obligaciones, y bajo NIIF contaban con $\$ 3,26$ por $\$ 1$ adeudado. Por otra parte, los indicadores de prueba ácida y de solidez bajo COLGAAP muestra que los fondos contaban a corto y largo plazo con $\$ 2,97$ y $\$ 3,70$ respectivamente por $\$ 1$ adeudado, y bajo NIIF mejoró a $\$ 3,03$ y $\$ 3,71$ respectivamente. Pero al hacer la modelación, según el indicador de solidez la capacidad de los fondos para cubrir sus obligaciones a corto y largo plazo desmejoró significativamente, disminuyendo a $\$ 1,31$ por cada peso que adeuda.

Sin embargo, los resultados finales de los índices del grupo de liquidez prácticamente no cambian por el efecto de la implementación de NIIF, ni tampoco en la respectiva modelación de NIIF FULL. 
REVISTA DE LA UNIVERSIDAD DEL ZULIA. 3época. Año 11 N² 29, 2020 Jaime Guevara et al. /// Convergencia a NIIF: impactos en estructura financiera ...

Tabla VI. Indicadores de Riesgo de Liquidez

\begin{tabular}{|c|c|c|c|c|c|c|c|}
\hline Indicador & Cálculo & Colgaap & $\begin{array}{c}\text { Umbral } \\
\text { Colgaap }\end{array}$ & NIIF & $\begin{array}{c}\text { Umbral } \\
\text { NIIF }\end{array}$ & $\begin{array}{c}\text { Modelación } \\
\text { NIIF }\end{array}$ & $\begin{array}{c}\text { Umbral } \\
\text { Modelación }\end{array}$ \\
\hline $\begin{array}{c}\text { Fondo de } \\
\text { Liquidez }\end{array}$ & $\begin{array}{c}\text { Disponible } \\
/ \text { Total } \\
\text { Depósitos }\end{array}$ & $5.18 \%$ & Verde & $5.18 \%$ & Verde & $5.18 \%$ & Verde \\
\hline $\begin{array}{c}\text { Nivel de } \\
\text { Disponible } \\
\text { e } \\
\text { Inversiones }\end{array}$ & $\begin{array}{c}\text { Inversiones } \\
/ \text { Total de } \\
\text { Activos }\end{array}$ & $12.01 \%$ & Verde & $12.03 \%$ & Verde & $12.03 \%$ & Verde \\
\hline
\end{tabular}

Fuente: Elaboración propia a partir de datos de fondos de empleados del depto. de Sucre que reportaron información de convergencia a NIIF para Pymes a la Superintendencia de economía solidaria. (La modelación se realizó tomando los aportes de capital como pasivos).

\subsection{Indicadores de Riesgo de Cartera}

De acuerdo a los indicadores de riesgo de cartera aplicados bajo COLGAAP, NIIF y modelación, los fondos de empleados en estudio se encuentran en el umbral AMARILLO en cuanto a la cobertura de provisión general, presentando una leve disminución debido al aumento de la cartera de créditos. La cobertura de inversión de ahorros arroja un aumento de 0,1 veces debido a los ajustes por convergencia en la cuenta créditos de consumo, otras garantías - con libranza y reclasificación de los intereses. Para los indicadores de Cartera el impacto de NIIF y modelación con referencia a COLGAAP no es evidente ya que el indicador queda en umbral VERDE en los tres escenarios y su variación no es significativa

Sin embargo, teniendo en cuenta los resultados del indicador de cobertura de provisión general, es necesario que los fondos hagan un replanteamiento de la recuperación de la cartera. 
REVISTA DE LA UNIVERSIDAD DEL ZULIA. $3^{\text {a }}$ época. Año 11 N²9, 2020 Jaime Guevara et al. /// Convergencia a NIIF: impactos en estructura financiera ...

Tabla VII. Indicadores de Riesgo de Cartera

\begin{tabular}{|c|c|c|c|c|c|c|c|}
\hline Indicador & Cálculo & Colgaap & $\begin{array}{c}\text { Umbral } \\
\text { Colgaap }\end{array}$ & NIIF & $\begin{array}{c}\text { Umbral } \\
\text { NIIF }\end{array}$ & $\begin{array}{c}\text { Modelación } \\
\text { NIIF }\end{array}$ & $\begin{array}{c}\text { Umbral } \\
\text { Modelación }\end{array}$ \\
\hline $\begin{array}{c}\text { Cobertura } \\
\text { Provisión } \\
\text { General }\end{array}$ & $\begin{array}{c}\text { Provisión } \\
\text { Cartera/Total } \\
\text { Cartera Bruta }\end{array}$ & $0.55 \%$ & Amarillo & $0.54 \%$ & Amarillo & $0.54 \%$ & Amarillo \\
\hline $\begin{array}{c}\text { Cobertura } \\
\text { de } \\
\text { inversión } \\
\text { de ahorros }\end{array}$ & $\begin{array}{c}\text { Cartera Bruta/ } \\
\text { Depósitos }\end{array}$ & 3.7 & Verde & 3.8 & Verde & 3.8 & Verde \\
\hline
\end{tabular}

Fuente: Elaboración propia a partir de datos de fondos de empleados del depto. de Sucre que reportaron información de convergencia a NIIF para Pymes a la Superintendencia de economía solidaria. (La modelación se realizó tomando los aportes de capital como pasivos).

\subsection{Indicadores de Riesgo Financiero}

El indicador de disminución patrimonial refleja una leve variación de COLGAAP a NIIF debido al aumento en los excedentes y/o pérdidas acumulados por adopción por primera vez. Pero en cuanto al resultado de la modelación que es de 59,1l siendo la variación significativa, lo anterior es debido principalmente a la reclasificación de los aportes sociales temporalmente restringidos al pasivo, sin embargo, no afecta el umbral en el que se encuentran los escenarios antes indicados, es decir, VERDE, posiblemente debido a que los fondos de empleados del departamento de Sucre, tienen suficientes recursos en otros rubros de patrimonio como son la cuenta de reservas con $\$ 768.3$ millones y fondos de destinación específica con $\$ 216.3$ millones, adicionalmente para el año de estudio los fondos obtuvieron resultados del periodo representativos dentro del total de patrimonio siendo de $\$ 350.3$ millones. Sin embargo, como parte de las estrategias de cada fondo, estos 
REVISTA DE LA UNIVERSIDAD DEL ZULIA. 3ㄹe época. Año 11 N² 29, 2020 Jaime Guevara et al. /// Convergencia a NIIF: impactos en estructura financiera ...

dineros pueden ser distribuidos en reservas fortaleciendo el componente patrimonial o se pueden usar en distintos beneficios para los asociados.

Por otra parte, el indicador de activo productivo muestra una leve disminución debido al aumento del activo por los ajustes mencionados en indicadores anteriores.

En resumen, los indicadores de riesgo financiero están en el umbral VERDE en los tres escenarios, lo que es favorable para estos fondos, sin mostrar impactos importantes por la convergencia a NIIF

\section{Tabla VIII. Indicadores de Riesgo Financiero}

\begin{tabular}{|c|c|c|c|c|c|c|c|}
\hline Indicador & Cálculo & Colgaap & $\begin{array}{c}\text { Umbral } \\
\text { Colgaap }\end{array}$ & NIIF & $\begin{array}{c}\text { Umbral } \\
\text { NIIF }\end{array}$ & $\begin{array}{c}\text { modelación } \\
\text { NIIF }\end{array}$ & $\begin{array}{c}\text { umbral } \\
\text { modelación }\end{array}$ \\
\hline $\begin{array}{c}\text { Disminución } \\
\text { patrimonial }\end{array}$ & $\begin{array}{c}\text { Patrimonio } \\
\text { / Capital } \\
\text { Social }\end{array}$ & 1.47 & Verde & 1.46 & Verde & 59.11 & Verde \\
\hline $\begin{array}{c}\text { Activo } \\
\text { productivo }\end{array}$ & $\begin{array}{c}\text { Activos } \\
\text { productibl } \\
\text { es/ Total } \\
\text { de Activos }\end{array}$ & $88 \%$ & Verde & $89 \%$ & Verde & $89 \%$ & Verde \\
\hline
\end{tabular}

Fuente: Elaboración propia a partir de datos de fondos de empleados del depto. de Sucre que reportaron información de convergencia a NIIF para Pymes a la Superintendencia de economía solidaria. (La modelación se realizó tomando los aportes de capital como pasivos).

\subsection{Indicadores de Riesgo de Estructura}

En los indicadores de riesgo de estructura aplicados bajo Colgaap, NIIF y modelación, existe variaciones importantes, debido a los ajustes por convergencia a NIIF y en la modelación de NIIF FULL, realizados en los rubros cartera de créditos y activos materiales, y en la cuenta aportes sociales temporalmente restringidos, el cálculo de 
REVISTA DE LA UNIVERSIDAD DEL ZULIA. 3época. Año 11 N² 29, 2020 Jaime Guevara et al. /// Convergencia a NIIF: impactos en estructura financiera ...

aportes sociales mínimos no reducibles, y la reclasificación de aportes sociales temporalmente restringidos, para el caso de la modelación.

En cuanto al indicador de Endeudamiento el resultado del umbral es VERDE tanto para Colgaap como NIIF, sin embargo, en el caso de la modelación el resultado del umbral es ROJO el cual desmejora por la reclasificación de los fondos sociales. En cuanto al indicador aporte social sobre activos, para los casos Colgaap y NIIF es ROJO, y en la modelación mejora sustancialmente, pasando al umbral VERDE. Debido principalmente a la maximización de los activos no circulantes.

En este conjunto de indicadores y especialmente en el de endeudamiento se ve muy afectado en el proceso de modelación por lo que se considera que fue adecuada la medida tomada por la Superintendencia de Economía Solidaria de dejar los aportes sociales temporalmente restringidos en el patrimonio.

\subsection{Indicadores de Riesgo de Endeudamiento}

El indicador de endeudamiento total muestra que los activos de los fondos están siendo financiados en un $27 \%$ con los pasivos, con la implementación de las NIIF esta financiación tiene una disminución debido a ajustes en cuentas del activo, mencionados en indicadores anteriores. Dicho indicador aumenta significativamente al hacer la modelación ubicándose en $76 \%$ por la reclasificación de los aportes sociales temporalmente restringidos al pasivo. En cuanto al indicador índice de propiedad se refleja un aumento, pasando de 72,94\% bajo Cogaap a 73,04\% bajo NIIF, como resultado del incremento del activo y de la cuenta excedentes y/o pérdidas acumuladas por adopción por primera vez, reflejando que el apalancamiento con el patrimonio es mayor. Caso contrario sucede con la modelación, donde el apalancamiento con patrimonio es de $24 \%$.

Teniendo en cuenta que el indicador de endeudamiento ideal debe estar situado entre el $40 \%$ y el $60 \%$, podemos decir que para los dos primeros escenarios los fondos pueden estar incurriendo en un exceso de capital ocioso, con la consiguiente pérdida de rentabilidad de sus recursos. Por el contrario, para el caso de la modelación el indicador de 
REVISTA DE LA UNIVERSIDAD DEL ZULIA. 3ㄹe época. Año 11 N² 29, 2020 Jaime Guevara et al. /// Convergencia a NIIF: impactos en estructura financiera ...

endeudamiento arroja un resultado mayor al 60\% lo que significa que los fondos están soportando un excesivo volumen de deuda.

Adicionalmente, destacamos que, con la modelación el apalancamiento de los activos se invierte, en el entendido de que bajo Colgaap y NIIF estaba mayormente financiado por el patrimonio, y bajo modelación son financiados mayormente por el pasivo.

Tabla IX. Indicadores de Riesgo de Estructura

\begin{tabular}{|c|c|c|c|c|c|c|c|}
\hline Indicador & Cálculo & Colgaap & Umbral & NIIF & Umbral & $\begin{array}{l}\text { Modelación } \\
\text { NIIF }\end{array}$ & $\begin{array}{l}\text { Umbral } \\
\text { modelación }\end{array}$ \\
\hline $\begin{array}{c}\text { Endeudamiento } \\
\text { sin Ahorro } \\
\text { Permanente }\end{array}$ & $\begin{array}{c}\text { Total } \\
\text { Pasivo - } \\
\text { Fondos } \\
\text { sociales + } \\
\text { Depósitos / } \\
\text { Total } \\
\text { Activo }\end{array}$ & $1.78 \%$ & Verde & $1.78 \%$ & Verde & $50.96 \%$ & Rojo \\
\hline $\begin{array}{c}\text { Depósitos } \\
\text { sobre Activos }\end{array}$ & $\begin{array}{c}\text { Depósitos / } \\
\text { Total } \\
\text { Activos }\end{array}$ & $23.09 \%$ & Rojo & $23.01 \%$ & Rojo & $23.01 \%$ & Rojo \\
\hline $\begin{array}{l}\text { Aporte Social } \\
\text { sobre Activos }\end{array}$ & $\begin{array}{c}\text { Aporte } \\
\text { Social / } \\
\text { Activo } \\
\text { Total }\end{array}$ & $49.77 \%$ & Rojo & $49.59 \%$ & Rojo & $1.22 \%$ & Verde \\
\hline $\begin{array}{c}\text { Capital } \\
\text { Institucional } \\
\text { sobre Activos }\end{array}$ & $\begin{array}{c}\text { Patrimonio } \\
\text { - Capital } \\
\text { Social) / } \\
\text { Activo } \\
\text { Total }\end{array}$ & $23.18 \%$ & Verde & $23.45 \%$ & Verde & $23.45 \%$ & Verde \\
\hline
\end{tabular}

Fuente: Elaboración propia a partir de datos de fondos de empleados del depto. de Sucre que reportaron información de convergencia a NIIF para Pymes a la Superintendencia de economía solidaria. (La modelación se realizó tomando los aportes de capital como pasivos). 
REVISTA DE LA UNIVERSIDAD DEL ZULIA. 3ㄹe época. Año 11 N² 29, 2020 Jaime Guevara et al. /// Convergencia a NIIF: impactos en estructura financiera ...

Tabla X. Indicadores de Riesgo de Endeudamiento

\begin{tabular}{|c|c|c|c|c|}
\hline Indicador & Cálculo & Colgaap & NIIF & $\begin{array}{c}\text { Modelación } \\
\text { NIIF }\end{array}$ \\
\hline $\begin{array}{c}\text { Endeudamiento } \\
\text { total }\end{array}$ & $\begin{array}{c}\text { Pasivo Total/ } \\
\text { Activo Total }\end{array}$ & $27.06 \%$ & $26.96 \%$ & $76.14 \%$ \\
\hline Indice de Propiedad & $\begin{array}{c}\text { Patrimonio / } \\
\text { Activo }\end{array}$ & $72.94 \%$ & $73.04 \%$ & $23.86 \%$ \\
\hline
\end{tabular}

Fuente: Elaboración propia a partir de datos de fondos de empleados del depto. de Sucre que reportaron información de convergencia a NIIF para Pymes a la Superintendencia de economía solidaria. (La modelación se realizó tomando los aportes de capital como pasivos).

\section{Conclusiones}

El contenido de la NIC 32 plantea un cambio en la forma tradicional de contabilización del capital social en las entidades de la economía solidaria, de acuerdo a la modelación realizada, este tratamiento contable de las aportaciones de los socios como pasivos en vez de patrimonio neto, aumenta el endeudamiento y deterioro de su solvencia, con efectos negativos en las calificaciones de riesgos, adicionalmente se afecta negativamente la estructura financiera de los fondos. Este aspecto es especialmente relevante para las grandes cooperativas, en las que tienen gran importancia los indicadores de solvencia o las calificaciones de las agencias. En este mismo sentido deduce que, debido a un incremento a causa de recibir los aportes sociales como pasivo exigible y pasar a convertirse en depósitos, estos causarán un mayor costo de interés por la prima de seguro de los depósitos, lo cual implica gastos que se verán reflejados en el Estado de Resultados y terminarán afectando los excedentes. Adicionalmente este tipo de entidades se van a 
REVISTA DE LA UNIVERSIDAD DEL ZULIA. 3ㄹe época. Año 11 N² 29, 2020 Jaime Guevara et al. /// Convergencia a NIIF: impactos en estructura financiera ...

encontrar en una situación de desventaja frente a otras formas de asociación cooperativa que desde su constitución cuentan con una determinada cifra de recursos propios.

Desde este punto de vista, consideramos acertada la decisión de la Superintendencia de Economía Solidaría de dejar los aportes sociales dentro del patrimonio modificando la NIC 32 y la IFRIC 2. Evitando impactos en su estructura y endeudamiento. Adicionalmente consideramos procedente recomendar que estas entidades, en su proceso de convergencia a NIIF, realicen una actualización de sus estatutos con el fin de adecuar y aumentar el capital mínimo irreducible, de tal manera que permitan o posibiliten que su estructura de financiación y, por tanto, sus posibilidades de operación y márgenes de liquidez no se vean afectadas duramente al disminuirse de manera considerable el patrimonio, vía traslado de aportes sociales a pasivos exigibles y el posterior retiro de estos. En definitiva, los fondos de empleados deben asumir el proceso de convergencia a NIIF con disciplina ya que es necesario ser muy precisos en la definición de sus políticas contables. Por lo que se infiere una vez analizados los diferentes indicadores para los tres escenarios, a manera de sugerencia para los diferentes fondos, como organismos de control y supervisión, es que los aportes sociales temporalmente restringidos permanezcan en el patrimonio como lo establece la normativa colombiana.

De otro lado el cambio de Colgaap a NIIF no se observan variaciones importantes en cuento a los indicadores de liquidez, cartera, riesgo financiero, estructura y endeudamiento ya que en los dos escenarios todos los indicadores se mantienen en el umbral verde, por lo que se infiere que no hubo ningún impacto por el cambio de política contable.

Ahora, a manera de ahondar aún más en la problemática, sería pertinente plantear una investigación del comportamiento de los entes pertenecientes al sector solidario, de los países latinoamericanos, en donde se socialice los resultados obtenidos como experiencia, de haber adoptado la NIC 32.

\section{Referencias}


REVISTA DE LA UNIVERSIDAD DEL ZULIA. 3época. Año 11 N²9, 2020 Jaime Guevara et al. /// Convergencia a NIIF: impactos en estructura financiera ...

Acuña, M. (2011). Crisis financiera internacional: rupturas y desafíos, Revista de la Universidad del Zulia, 2 (4), 39-58.

Álvarez B. y Suarez E. (2015). Calificación de instrumentos financieros en las sociedades cooperativas a raíz de la NIC 32. La solución española. Innovar Vol.25 pp. 9-20

Arias, M y Sánchez A. (2014) Bases de medición: correspondencia entre las Normas Internacionales de Información financiera, los Estándares Internacionales de Valuación y el contexto actual colombiano. Cuadernos de contabilidad. Vol. 15 No 37 pp. 25-37

Barth, M., \& Landsman, W. (2008). International Accounting Standards and Accounting Quality. Journal of Accounting Research, Vol 46. No.3 pp. 467-498.

Callao, S., Ferrer, C., Jarne, J. y Laínez, J. (2010). IFRS Adoption in Spain and the United Kingdom: Effects on Accounting Numbers and Relevance.

Cañibano, L. y Gisbert, A. (2007). El proceso de armonización contable internacional, la estrategia europea y la adaptación de la normativa contable en España. Contaduría Universidad de Antioquia, 5l, pp 11-40.

Cardozo, H. (2008), Fondos de Empleados, Ecoe ediciones, primera edición.

Cardozo, H. (2015), Contabilidad de entidades de economía solidaria bajo NIIF para Pyme, Ecoe ediciones, quinta edición.

Castaño, C. y Arias, J. (2013), Análisis Financiero Integral de Empresas Colombianas 20092010; Perspectivas de Competitividad Regional, Entramado, vol. 9, núm 1.

Castaño, C., Zamarra, J. y Correa, J., (2014) Efectos financieros en una cooperativa colombiana por la implementación de normas internacionales de contabilidad financiera para las pymes en su balance de apertura. Cuadernos de Contabilidad. Vol 15 No 35 pp. 427459

Cenicoop y Confecoop. (2014) Desempeño sector cooperativo colombiano 2014.

Cerda, H. (2002). Los elementos de la investigación. Cómo reconocerlos, diseñarlos y construirlos. Bogotá D.C.: El Búho.

Circular Externa No. 08, emitida por la Superintendencia de la economía solidaria, sobre el asunto: solicitud de información financiera proceso de convergencia a NIIF. 27 de febrero de 2015.

Colombia (1988). Ley 79 de 1988, por el cual se actualiza la legislación cooperativa. Diario Oficial,38.648, 23 de diciembre de 1988. 
REVISTA DE LA UNIVERSIDAD DEL ZULIA. 3ㄹe época. Año 11 N² 29, 2020 Jaime Guevara et al. /// Convergencia a NIIF: impactos en estructura financiera ...

Colombia (1989). Decreto 1481 de 1989, por el cual se determinan la naturaleza, características, constitución, regímenes internos de responsabilidad y sanciones, y se dictan medidas para el fomento de los fondos de empleados. Diario Oficial, 7 de Julio de 1989.

Colombia (1998). Ley 454 de 1998, por la cual se determina el marco conceptual que regula la economía solidaria, se transforma el Departamento Administrativo Nacional de Cooperativas en el Departamento Administrativo Nacional de la Economía Solidaria, se crea la Superintendencia de la Economía Solidaria, se crea el Fondo de Garantías para las Cooperativas Financieras y de Ahorro y Crédito, se dictan normas sobre la actividad financiera de las entidades de naturaleza cooperativa y se expiden otras disposiciones. Diario Oficial, 43.357, 6 de agosto de 1998.

Colombia (2004). Decreto 186 de 2004, por el cual se modifica la estructura de la Superintendencia de la Economía Solidaria. Diario Oficial, 45443, 27 de enero de 2004.

Colombia (2015). Decreto Único Reglamentario 2420, por medio del cual se expide el Decreto Único Reglamentario de las Normas de Contabilidad, de Información Financiera y de Aseguramiento de la Información y se dictan otras disposiciones. Diario Oficial, 49.726, 14 de diciembre de 2015.

Colombia (2015). Decreto 2496, por medio del cual se modifica el Decreto 2420 de 2015 Único Reglamentario de las Normas de Contabilidad, de Información Financiera y de Aseguramiento de la Informacióny se dictan otras disposiciones. 23 de diciembre de 2015.

Correa, J., Castaño, C. y Ramírez, L.. (2010). Análisis financiero integral: elementos para el desarrollo de las organizaciones. En: Lúmina. $N^{\circ} 11$. (2010), pp. 188.

Correa, J. y Ojeda, N. (2014). Impactos financieros y sociales de la aplicación de las NIIF sobre instrumentos financieros en las Cooperativas de Ahorro y Crédito en el Área Metropolitana del Valle de Aburrá. Universidad de Antioquia.

Cubedo, M. (2007). El régimen económico de las sociedades cooperativas: situación actual y apuntes para una reforma. Universitat de València.

Dasker, H., Hail, L., Leuz, C., \& Verdi, R. (2008). Mandatory IFRS Reporting around the World: Early Evidence on the Economic Consequences. Journal of Accounting Research, 46(5), pp 1085-1142.

García, O. (1999). Administración Financiera, Prensa Moderna Impresores S.A., Tercera edición.

Gómez, M. (2014). Una evaluación del enfoque de las Normas Internacionales de Información Financiera (NIIF) desde la teoría de la contabilidad y el control. Innovar, revista de ciencias administrativas y sociales. No 24 pp. 112-131 
REVISTA DE LA UNIVERSIDAD DEL ZULIA. 3época. Año 11 N² 29, 2020 Jaime Guevara et al. /// Convergencia a NIIF: impactos en estructura financiera ...

International Accounting Standards Board, IASB (2010). Marco conceptual de IFRS. International Accounting Standards Board, IASB (2011). International Financial Reporting Standards. IFRS 9, IAS 39, IAS 16, IAS 1.

International Accounting Standards Board, IASB (2009). IFRS for SMEs. Versión en español.

Legis editores S.A. (2015), primera edición.

Lima, R. (2007). Información financiera para pymes. Revista del Centro de Investigación de la Universidad de la Salle, 27 (7), pp. 67-75.

Martínez, F. (2014). Modelo de adaptación de las Normas Internacionales de Información Financiera a la estructura patrimonial de las cooperativas panameñas. Facultad de Administración de Empresas y Contabilidad Dpto. Contabilidad Financiera Universidad de Panamá. Gestión Joven Revista de la Agrupación Joven Iberoamericana de Contabilidad y Administración de Empresas (AJOICA), N 12-2014, pp. 15-30.

Molina, R. (2007). La reforma de la legislación mercantil en materia contable. Una breve referencia a su incidencia en las cooperativas y sociedades laborales. CIRIEC España, Revista de Economía Pública, Social y efectos de la NIIF para pyme.

Morales, M. L. (2014). Factores Claves de Éxito en la Gestión Administrativa de las Entidades Cooperativas Especializadas de Ahorro y Crédito. Universidad Autónoma de Occidente.

Pérez, R., Urquía, E. y Muñoz, C. (2009). ¿Las pymes españolas tienen interés por las NIIF? Partida Doble, 215, pp. 20-26.

Polo, F. (2007). Impactos de las normas internacionales de información financiera en el régimen económico de las sociedades cooperativas. CIRIEC-España, Revista de Economía Pública, Social y Cooperativa, pp 58, 83-108.

Rendón, B., Montaño, E. y Gaitán, G. (2013). Las NIIF y su impacto en las cooperativas en Colombia a diciembre 31 de 2011. Cuadernos de contabilidad Vol. 29 No 50 pp. 176-185

Rendón, B., Rodríguez, J. y Riascos, P. (2013). Análisis del impacto en el capital institucional de las cooperativas de ahorro y crédito frente a la aplicación de las NIIF. Caso cooperativas de ahorro y crédito del Valle del Cauca a diciembre 31 de 2012. Según clasificación COLCIENCIAS Artículo de investigación científica y tecnológica. Contaduría Pública de la Universidad del Valle.

Restrepo, J. y Londoño, M. L. (2011). Colombia: hacia la adopción y aplicación de las NIIF y su importancia. Adversia, 8. 
REVISTA DE LA UNIVERSIDAD DEL ZULIA. 3ㄹ época. Año ll N²9, 2020 Jaime Guevara et al. /// Convergencia a NIIF: impactos en estructura financiera ...

Silva, B. y Garrido, C. (2006). La convergencia a las normas internacionales de información financiera en Chile. Capic Review, 4, pp. 9-19.

Superintendencia de la Economía Solidaria, Supersolidaria (2010). Circular básica contabley financiera, modificación del capítulo XVI de la circular contable y financiera 004 de 2008.

Torres, F. y Rodríguez, B. (2008). La maximización de los beneficios bajo los estándares de contabilidad financiera: una aproximación a las aplicaciones en la PYME. Cuadernos de Contabilidad, 4 (24), pp 73-130.

Vargas Vasserot, C. (2007). Los previsibles efectos de la NIC 32 en el sector cooperativo. Revista de Estudios Cooperativos, 91(1), pp 120-159

Vásquez, N. (2013). Impacto de las IFRS en los reportes financieros de empresas listadas en la Bolsa Mexicana de Valores. Revista de Administración, Finanzas y Economía, 1 (6), pp. 89107.

Vásquez, N., Carril, M. y Pascual, M (2013). Estado actual de Latinoamérica en relación a su proceso de adopción de las NIIF. Contabilidad e Auditoría, Investigaciones en teoría contable, 19 (38), pp. 55-104.

Yáñez, V., Pilar, F. e Inostrosa, C. (2010). Impacto de las IFRS en la valoración de empresas: evidencia para una muestra de empresas chilenas cotizadas.

Zapata, G. y Hernández, A. (2010). Reflexiones sobre la aplicación de las NIIF en las pymes venezolanas. Compendium, 13 (25),pp. 23-41. 